\title{
Uma Proposta para o Ensino de Matrizes com o Apoio de Tecnologia
}

\author{
Elizandro Max Borba, Vilmar Trevisan, \\ Departamento de Matemática Pura e Aplicada, PPGMAp, UFRGS \\ Av. Bento Gonçalves, 9500 - Prédio 43111 - Agronomia \\ 91509-900, Porto Alegre, RS \\ E-mail: elizandro.max@ufrgs.br
}

\begin{abstract}
Resumo: Atualmente, o ensino de matrizes tende a enfatizar o uso de matrizes pequenas, adequadas para o cálculo manual. Este trabalho propõe uma abordagem baseada em tecnologia, usando recursos de informática como planilhas eletrônicas e applets disponíveis na Internet, a fim de permitir a exploração de matrizes maiores, além o uso do Algoritmo de Escalonamento para resolver Sistemas Lineares, e de conexão com a Teoria dos Grafos, com aplicações reais para cada um desses tópicos. Usamos a Abordagem Incorporada como principal ferramenta teórica.
\end{abstract}

\section{Introdução}

No Ensino Médio, tende-se a restringir a manipulação de matrizes (e consequentemente a resolução de sistemas lineares) a matrizes pequenas, que permitam o cálculo no papel. Mesmo matrizes simples, como as quadradas de ordem 3, exigem cálculos tediosos e sujeitos a erros, ainda que se restrinja os valores a números inteiros ou frações simples. As matrizes maiores normalmente são apenas referenciadas, de maneira um tanto distante, como exemplo de aplicação de resultados mais gerais.

Atualmente, a Álgebra Linear tem papel fundamental na Matemática Aplicada. Várias aplicações modernas podem ser modeladas com o emprego de matrizes e sistemas lineares: balanceamento de equações químicas, design de circuitos elétricos, computação gráfica, controle de tráfego, sistemas de navegação, interação entre setores da economia, entre muitas outras. Entretanto, mesmo sistemas relativamente simples exigem a manipulação de matrizes grandes o suficiente para inviabilizar, em termos práticos, o cálculo no papel.

Hoje em dia, o acesso a recursos de informática é mais amplo do que em qualquer período na história. Existem inúmeras ferramentas computacionais que podem auxiliar na manipulação de matrizes. Assim, não há porque limitar o contato do aluno a matrizes $3 \times 3 \mathrm{com}$ números inteiros. As ferramentas computacionais disponíveis possibilitam maior agilidade nas manipulações, o uso de valores mais realísticos, e uma melhor apreciação dos processos que estão por trás da resolução dos sistemas. Com isso, abre-se o caminho para poder experimentar as matrizes e sistemas lineares em contextos mais próximos da realidade e mais interessantes.

O objetivo foi fazer uma análise da abordagem costumeira sobre o assunto, pesquisar sobre aplicações que possam ser apresentadas no Ensino Médio, com as adaptações necessárias, e então elaborar atividades que contemplem o uso das ferramentas computacionais já citadas. A elaboração das atividades usa a Abordagem Incorporada como principal ferramenta teórica. Essas atividades foram implementadas em um disciplina eletiva no Colégio de Aplicação da UFRGS (CAp), oferecida a alunos do segundo ano do Ensino Médio.

\section{Justificativa}

Desde o surgimento dos computadores eletrônicos, em meados do século 20, o estudo de matrizes tem estado cada vez mais ligado às suas aplicações computacionais, envolvendo matrizes de tamanho muito maior que as abordadas usualmente. Entretanto, os livros didáticos geralmente não contemplam o uso de ferramentas computacionais para esse tópico. Buscamos, portanto, elaborar uma proposta no sentido de tentar diminuir essa discrepância. 
Em sua versão mais recente, os PCN [2] afirmam que o estudo de sistemas lineares deve "receber um tratamento que enfatize sua importância cultural, isto é, estender os conhecimentos que os alunos possuem (...) sobre a resolução de sistemas de duas equações e duas incógnitas para sistemas lineares 3 por 3, aplicando esse estudo à resolução de problemas simples de outras áreas do conhecimento. Uma abordagem mais qualitativa e profunda deve ser feita dentro da parte flexível do currículo, como opção específica de cada escola."

Entendemos que essa predileção por "problemas simples" e sistemas pequenos seja devida à premissa de que os cálculos serão necessariamente manuais. $\mathrm{O}$ uso do computador, como já frisamos, seria a ferramenta que possibilitaria a extensão das aplicações para problemas "mais complexos". Entretanto, os PCN parecem não vislumbrar a totalidade do potencial computador como ferramenta de automação dos cálculos, tratando-o principalmente como ferramenta de edição, comunicação e busca. Além disso, a possibilidade de efetivamente utilizar a internet para realizar cálculos, como pretendemos fazer em alguns momentos, é algo que escapa à visão apresentada nos últimos PCN. Acreditamos que isso deva ser corrigido, pois no ritmo em que ocorrem as revoluções tecnológicas hoje em dia, é difícil escrever alguma linhaguia sobre informática que continue atual por muito tempo.

Apesar das ressalvas já feitas, nota-se que os elaboradores dos PCN têm ciência de que a popularização do computador leva a novos paradigmas didáticos. Entretanto, isso não se reflete nos livros didáticos. Algumas aplicações interessantes de sistemas lineares são mostradas em alguns livros, como exemplos simples de controle de fluxo de trânsito e balanceamento de equações químicas, citadas acima, e alocação de tarefas. Em nenhum caso, entretanto, o limiar de três equações e três incógnitas é ultrapassado. Acreditamos ser esta a situação dominante. Outra evidência vem de Battaglioli [1], que analisa a abordagem a sistemas lineares em três livros didáticos de Ensino Médio, e conclui que "nenhum dos livros analisados apresenta alguma atividade com o uso do computador envolvendo sistemas lineares".

\section{Metodologia}

A principal ferramenta teórica utilizada no trabalho foi a abordagem incorporada, apresentada por Tall [4] [5]. Essa abordagem defende, em suma, que o caminho para os níveis mais abstratos do pensamento matemático é facilitado se passar por uma fase de interação visual e enativa, usando organizadores genéricos para manipular raízes cognitivas. Um organizador genérico é um ambiente (ou micromundo) que permite que o aprendiz manipule exemplos e (se possível) contraexemplos de um conceito matemático específico ou um sistema de conceitos relacionados. Uma raiz cognitiva é um conceito que (potencialmente) já possui significado para o estudante, e ainda contém as sementes de expansão cognitiva e posterior desenvolvimento teórico.

Assim, uma etapa importante na construção das atividades foi elencar candidatos a raízes cognitivas e organizadores genéricos para os diversos proceitos. Os organizadores genéricos utilizados serão recursos de software de dois tipos principais: planilhas eletrônicas e Applets. Nessas escolhas, preocupamo-nos com a portabilidade das atividades, ou seja, esperamos que elas possam ser reproduzidas com um número mínimo ou nulo de mudanças na plataforma de hardware e software onde as atividades forem porventura aplicadas.

\section{Concepção Da Prática}

Como o campo é muito amplo, foi necessário inicialmente limitar o escopo do trabalho antes de iniciar a confecção das atividades. Deixaremos de lado, por exemplo, temas como representação gráfica matricial e vetorial, transformações geométricas e aplicações de determinantes. Foram escolhidos os seguintes temas: (i) definição de matrizes por fórmulas, (ii) sistemas lineares e (iii) grafos. A seguir, para cada um desses temas, buscamos escolher uma raiz cognitiva e um organizador genérico. Discorreremos brevemente sobre os dois primeiros temas, e focaremos mais em grafos, que foi onde a questão da modelagem matemática ficou mais evidente. Finalmente, pesquisamos por aplicações que servissem de motivações para a confecção das atividades. 
Definição de matrizes por fórmulas: Um caminho bastante usado para o ensino de matrizes é o de definir uma matriz $A_{i \times j}$ por uma fórmula do tipo $a_{i j}=f(i, j)$. Podemos automatizar a geração dessa matriz com uma planilha eletrônica como o Excel. Com poucos cliques, pode-se expandir a matriz para um tamanho bem maior que o comumente usado nos exercícios desse tipo. No Excel 2010, a ferramenta de Formatação Condicional provê colorização automática conforme o valor da célula, criando um mapa análogo a um mapa de contorno colorizado. Essa visualização é, portanto, uma raiz cognitiva para a geração de matrizes, e o Excel, o organizador genérico associado. A tendência é que, com o tempo, essa funcionalidade de colorização automática, como tantas outras, torne-se comum em planilhas não comerciais.

Resolução de sistemas lineares: Nesse item, nosso objetivo foi inserir o escalonamento como método primário de resolução. O problema do escalonamento manual, como bem sabem os estudantes de Álgebra Linear em nível universitário, é o excesso de operações a serem executadas; e nessas operações, qualquer erro, como o esquecimento de um sinal, pode propagar-se de forma desastrosa nos passos posteriores. Queremos usar a eliminação gaussiana como raiz cognitiva, mas precisamos de uma ferramenta (organizador genérico) que execute as operações de linha, sendo que o aluno deve meramente indicá-las. Dessa forma, o aluno consegue ter uma visão melhor do processo de eliminação; outra vantagem é que os passos são todos reversíveis. Existem diversos applets e programas que executam operações de linha em uma matriz dada, como por exemplo o applet Row Echelon Form, localizado em um site (http://www.mathresource.iitb.ac.in/linear\%20algebra/RowEchelonForm) do IITB (Indian Institute of Technology Bombay).

Grafos: Grafos são objetos relativamente fáceis de explicar e de bom apelo visual - isso sem falar em suas aplicações computacionais, que os ligam intimamente às matrizes. Assim, é de se admirar que sejam tão pouco trabalhados no Ensino Básico. A inserção do ensino de grafos no Ensino Médio foi abordada por Malta [3], que destaca "a necessidade de representar um grafo de maneira que pudesse ser tratada ou processada no computador". Embora este trabalho realize várias atividades envolvendo a representação de grafos por matrizes de adjacência, não chega efetivamente a utilizar o computador para processá-las. Entretanto, admite que "a questão operatória (...) pode se tornar extensa quando um grafo tiver muitos vértices. Não chegamos a trabalhar com uma planilha de cálculo, mas talvez neste momento pudéssemos ter feito relação com algum software que facilitasse os cálculos." Tentaremos aqui preencher essa lacuna. Entretanto, antes de manipular as matrizes, é preciso que o aluno compreenda a representação do grafo como matriz de adjacência. Esta possivelmente será nossa escolha mais ousada de raiz cognitiva, por ser uma noção latente bastante abstrata.

O organizador escolhido foi um applet (que designaremos "applet 1") em um site (http://oneweb.utc.edu/ Christopher-Mawata/petersen/lesson7.htm) da UTC (University of Tennesee Chattanooga). Uma interessante abordagem é usada: é possível ver as mudanças na matriz à medida que o grafo é criado e alterado, como mostra a Figura 5. Entre as limitações estão a impossibilidade de inserir laços, traçar mais de uma aresta entre dois nós, ou criar arestas orientadas.

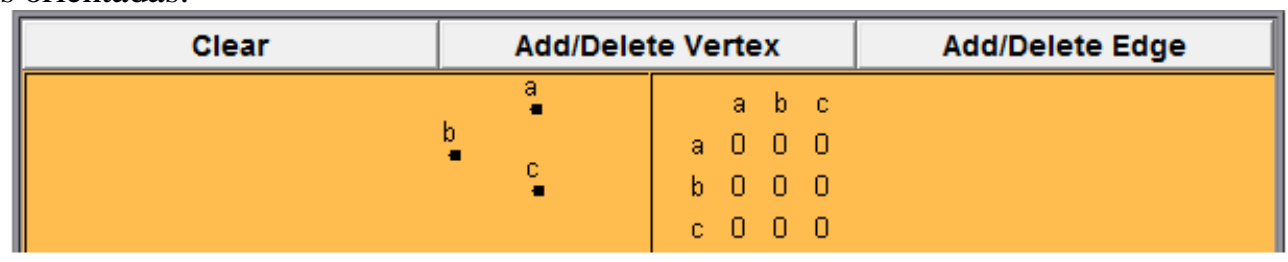

$\stackrel{a}{b} \quad \begin{array}{lllll}a & a & b & c & d \\ b & 0 & 0 & 0 & 0 \\ c & 0 & 0 & 0 & 0 \\ d & 0 & 0 & 0 & 0\end{array}$

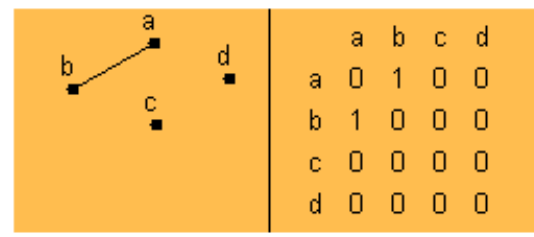

Figura 1: Telas do applet 1. 
No mesmo site, há outro applet interessante (“applet 2"), que pode ser útil na questão, também abordada por Malta, de encontrar caminhos entre nós através de potências da matriz de adjacência. Em uma única visualização, pode-se visualizar a matriz de adjacência $A$, e a matriz $A^{2}$, em que cada elemento $a_{i j}$ mostra o número de caminhos de tamanho 2 entre os nós $i$ e $j$, como mostrado na Figura 6. A partir daí, pode-se utilizar uma planilha eletrônica ou outra ferramenta para calcular potências maiores de $A$.

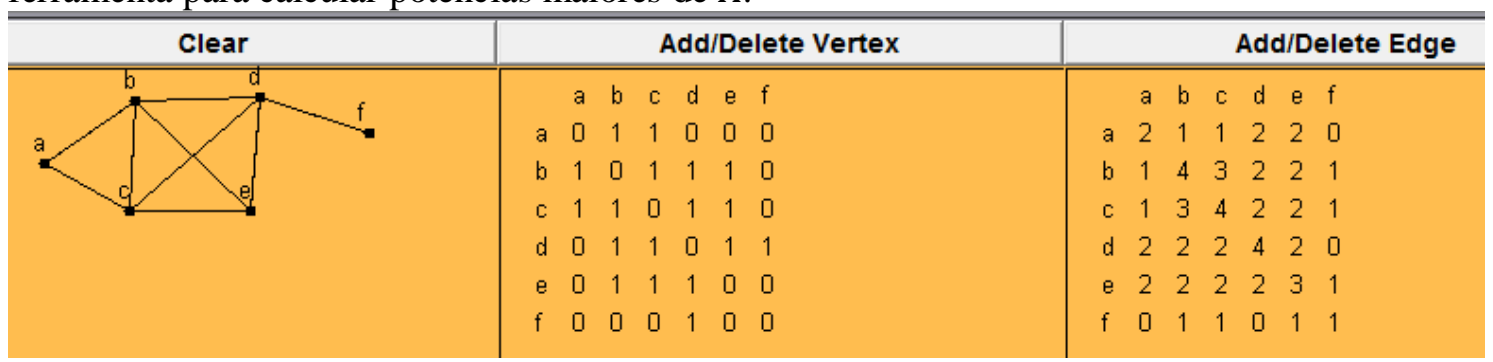

Figura 2: Tela do applet 2.

Os applets 1 e 2 tratam apenas da "ida" do proceito, ou seja, a construção da matriz a partir do grafo; a "volta", comparativamente mais abstrata, é a construção do grafo a partir da matriz. É digno de nota um applet ("applet 3") localizado em um site (http://www.cs.rpi.edu/research/groups/pb/graphdraw/headpage.html) do RPI (Rensselaer Polytechnic Institute) que traça grafos a partir de vários tipos de representações (incluindo matriz de adjacência), como mostrado na Figura 6. Entretanto, o método de entrada, em texto puro, não é tão adequado aos nossos propósitos, e houve problemas de compatibilidade com alguns navegadores. No presente trabalho, seu uso será apenas ilustrativo, sem ser empregado em atividades executadas pelos alunos.

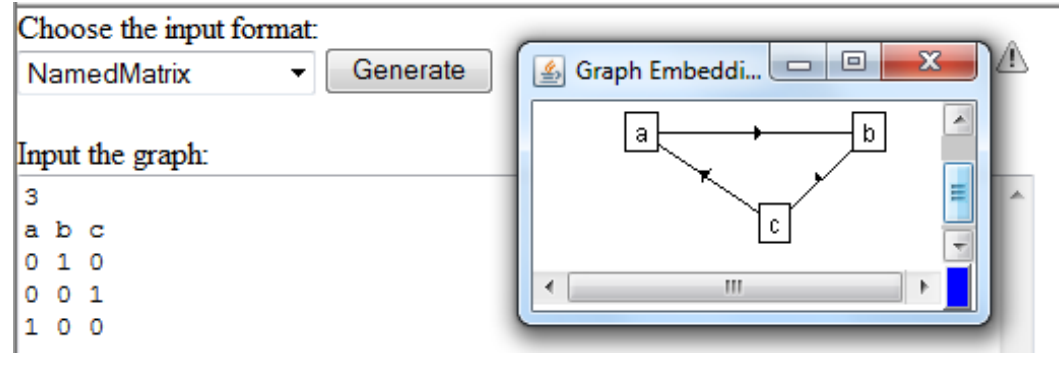

Figura 3: Tela do applet 3.

\section{Prática}

As atividades foram implementadas no Colégio de Aplicação (CAp) da UFRGS, na forma de uma disciplina eletiva chamada "Explorando Matrizes e Grafos com o Auxílio do Computador". Quinze alunos estavam inscritos. Neste capítulo, será feito um relato de algumas das atividades realizadas no decurso da disciplina.

Um componente vital do trabalho foi o site construído para a disciplina (https://sites.google.com/site/explorandomatrizesegrafos), que funcionou como guia para as atividades. O site foi hospedado pelo Google Sites, que permite a criação de sites de forma gratuita e de e que possibilita edição fácil e rápida - muitas vezes, a edição foi feita em plena aula. No site, foram criadas páginas para cada aula, contendo direcionamentos para as atividades a serem executadas, bem como registros a posteriori das atividades, resumos de conteúdos e gabaritos de cada aula, além de links para as ferramentas utilizadas. O site ficará disponível de forma permanente e livre.

As primeiras atividades desenvolvidas envolveram primariamente resolução de sistemas lineares sobre situações semirreais, como a distribuição de temperaturas em uma placa e balanceamento de equações químicas. Para essas, utilizamos o applet Row Echelon Form, que 
nomeamos "Ferramenta de Escalonamento de Matrizes". Como já fora mencionado, aprofundaremos mais a atividade envolvendo grafos.

Primeiramente, foi feita uma exposição sobre grafos, seus elementos e a matriz de adjacência $(A)$ e o significado de $A^{2}$, através do applet 2 citado no item 4.3. Posteriormente, foi proposta uma atividade com as seguintes etapas: (i) procurar um mapa na Internet (sugeriu-se acessar o Google Maps e mapear um conjunto de cidades, ou então algumas ruas de uma região qualquer, como os arredores da casa do aluno); (ii) escolher um conjunto de pontos desse mapa, e reproduzir o gráfico correspondente no applet 2 (por nós nomeada "Ferramenta de Criação de Grafos"), e identificar o que representam os pontos ( $a$ até $j$ ) do grafo; escolher dois pontos razoavelmente distantes, e descobrir a distância mínima entre eles, usando potências da matriz de adjacência $A$. Segue um exemplo de grafo criado por uma aluna, representando ruas nos arredores de sua casa.

(2)

Figura 4: Grafo criado por aluna (esquerda), sua matriz de adjacência $A$ (centro) e $A^{2}$ (esquerda).

Foi pedido então que, através da análise das potências de $A$, fosse descoberto o tamanho do caminho mínimo entre os pontos $a$ e $g$. Na própria ferramenta podemos ver que, como tanto em $A$ como $A^{2}$ o cruzamento entre $a$ e $g$ é 0 , então não há caminho de tamanho 1 ou 2.

Foi utilizada o "Matrices: Arithmetic" (http://www.quickmath.com/webMathematica3/quickmath/matrices/arithmetic/basic.jsp). Por limitações da Ferramenta de Criação de Grafos, a cópia de $A$ para esta página teve de ser feita manualmente. Ao obter $A^{3}$, verifica-se que ainda não há caminho de tamanho 3. Já a matriz $A^{4}$ indica que existem dois caminhos de tamanho 4.

\section{Considerações Finais}

$\mathrm{O}$ uso das ferramentas computacionais permitiu o rompimento da barreira representada pelos sistemas lineares $3 \times 3$, citada pelos $\mathrm{PCN}$ e seguida pelos livros didáticos. Além disso, foi possível usar valores que não precisam ficar restritos a valores "bem-comportados". A introdução do algoritmo de escalonamento foi bem assimilada pelos alunos. Outras ferramentas disponíveis na Internet permitem a manipulação de matrizes maiores, possibilitando a análise de modelos usando conceitos da Teoria dos Grafos.

Sabemos, entretanto, que as ferramentas têm muitas limitações. A tendência, entretanto, é que seja apenas questão de tempo para que essas ferramentas sejam melhoradas e surjam outras, com novas características interessantes, e também em português. Nem consideramos aqui as possibilidades que certamente surgirão com as novas tecnologias que emergem a cada instante, com novas formas de interação, como o uso de gestos em telas sensíveis a toque.

Didaticamente, os recursos computacionais desempenham um duplo papel positivo. Por um lado, facilitam a compreensão dos conceitos, através da abordagem incorporada. Por outro, auxiliam na resolução dos passos repetitivos. Os alunos que já haviam tido algum conteúdo de matrizes relatavam apenas lembrar que era preciso fazer uma grande quantidade de contas para montar uma matriz ou calcular um determinante, por exemplo. Liberado desse fardo, o aluno tem mais liberdade para visualizar o conteúdo de um ponto de vista mais elevado, facilitando a conexão com problemas reais, mais motivadores. Também pode-se fazer extensões para 
conteúdos mais avançados. Apenas a inclusão da análise de grafos já se constituiu em uma interessante inserção.

Talvez a implementação dessas atividades, com os recursos recomendados, seja quase utópica para a maioria das salas de aula brasileiras. Porém, a expectativa é que esse cenário mude aos poucos, como atestam iniciativas como o projeto UCA. Além disso, acreditamos que estudos como este podem servir de argumento para a aquisição de tais recursos, e como norteadores para sua utilização.

\section{Referências}

[1] Battaglioli, Sistemas Lineares na segunda série do Ensino Médio: um olhar sobre os livros didáticos. São Paulo, 2008.

[2] Brasil, Orientações Curriculares de Ensino Médio. Ciências da Natureza, Matemática e suas Tecnologias: Orientações Educacionais Complementares aos Parâmetros Curriculares Nacionais. Brasília: MEC, Secretaria da Educação Básica, 2006.

[3] Malta, Grafos no Ensino Médio: uma inserção possível. Porto Alegre: PPGEM da UFRGS, 2008.

[4] Tall, Using Technology to Support an Embodied Approach to Learning Concepts in Mathematics. História e Tecnologia no Ensino da Matemática , 1, 1-28, 2003.

[5] Tall e Vinner, Concept image and concept definition in mathematics, with special reference to limits and continuity. Educational Studies in Mathematics , 12, 151-169, 1981. 